Vol. 30, Issue 6, June 2007

Editorial

291 Pulmonary Alterations During BEP? Always Be Aware Of BIP

Sleijfer, S. (Rotterdam)

Original Articles

295 Measurement of DPD and TS Transcripts Aimed to Predict Clinical Benefit from Fluoropyrimidines: Confirmation of the Trend in Russian Colorectal Cancer Series and Caution Regarding the Gene Referees

Iyevleva, A.G.; Buslov, K.G.; Togo, A.V.; Matsko, D.E.; Filimonenko, V.P.; Moiseyenko, V.M.; Imyanitov, E.N. (St. Petersburg)

303 Quantitative and Semiquantitative Evaluation of Erythropoietin-Induced Bone Marrow Signal Changes in Lumbar Spine MRI in Patients with Tumor Anemia Ghanem, N.; Lerche, A.; Lohrmann, C.; Altehoefer, C.; Henke, M.; Langer, M. (Freiburg i.Br.)

311 Reliable Identification of Small Cell Lung Cancer in Cytological Specimens by Immunocytology Metzgeroth, G.; Mantz, C.; Kuhn, C.; Schultheis, B.; Hehlmann, R.; Hastka, J. (Heidelberg)

Clinical Cases

316 BRCA1 Germline Mutation in a Woman with Metaplastic Squamous Cell Breast Cancer Breuer, A. (Wiesbaden); Kandel, M. (Wiesbaden/Boston); Fisseler-Eckhoff, A. (Wiesbaden); Sutter, C. (Heidelberg); Schwaab, E.; Lück, H.-J.; du Bois, A. (Wiesbaden)

320 Increased Pulmonary FDG Uptake in BleomycinAssociated Pneumonitis

von Rohr, L.; Klaeser, B.; Joerger, M.; Kluckert, T.; Cerny, T.; Gillessen, S. (St. Gallen)

324 Concomitant Occurrence of Facial Cutaneous and Parotid Gland Metastases from Rectal Cancer after Pre-Operative Chemo-Radiotherapy

Nasti, G.; Facchini, G.; Caraglia, M.; Franco, R.; La Mura, A.; Staiano, M.; Budillon, A.; Iaffaioli, R.V.; Ottaiano, A. (Naples)
Band 30, Heft 6, Juni 2007

Editorial

291 Pulmonale Veränderungen unter BEP? Denken Sie immer auch an BIP

Sleijfer, S. (Rotterdam)

Originalarbeiten

295 Messung des DPD- und TS-Gehalts zur Vorhersage des klinischen Vorteils einer Fluoropyrimidintherapie: Bestätigung des Trends in einer russischen Kolorektalkarzinomserie und Warnung bezüglich der Kontrollgene Iyevleva, A.G.; Buslov, K.G.; Togo, A.V.; Matsko, D.E.; Filimonenko, V.P.; Moiseyenko, V.M.; Imyanitov, E.N. (St. Petersburg)

303 Quantitativer und semiquantitativer Nachweis von erythropoetininduzierten Veränderungen der Signalintensität des Knochenmarks in der MRT der Lendenwirbelsäule bei Patienten mit Tumoranämie Ghanem, N.; Lerche, A.; Lohrmann, C.; Altehoefer, C.; Henke, M.; Langer, M. (Freiburg i.Br.)

311 Zuverlässige Identifizierung kleinzelliger Lungenkarzinome in zytologischen Präparaten mittels Immunzytologie Metzgeroth, G.; Mantz, C.; Kuhn, C.; Schultheis, B.; Hehlmann, R.; Hastka, J. (Heidelberg)

Kasuistiken

316 BRCA1-Keimbahnmutation bei einer Patientin mit metaplastischem Plattenepithelkarzinom der Mamma Breuer, A. (Wiesbaden); Kandel, M. (Wiesbaden/Boston); Fisseler-Eckhoff, A. (Wiesbaden); Sutter, C. (Heidelberg); Schwaab, E.; Lück, H.-J.; du Bois, A. (Wiesbaden)

320 Erhöhte pulmonale FDG-Aufnahme bei Bleomycininduzierter Pneumonitis

von Rohr, L.; Klaeser, B.; Joerger, M.; Kluckert, T.; Cerny, T.; Gillessen, S. (St. Gallen)

324 Gleichzeitiges Auftreten von Metastasen eines Rektumkarzinoms in Gesichtshaut und Speicheldrüse nach präoperativer Chemoradiotherapie Nasti, G.; Facchini, G.; Caraglia, M.; Franco, R.; La Mura, A.; Staiano, M.; Budillon, A.; Iaffaioli, R.V.; Ottaiano, A. (Naples)

\begin{tabular}{ll}
\hline KARGER & @ 2007 S. Karger GmbH, Freiburg \\
Fax +497614520714 & Artikel (Volltext) und Inhaltsverzeichnisse \\
$\begin{array}{l}\text { E-mail Information@Karger.de } \\
\text { www.karger.com }\end{array}$ & sowie das vorläufige Inhaltsverzeichnis des nächsten Heftes: \\
www.karger.com/onk_bk.htm
\end{tabular}




\section{Vol. 30, Issue 6, June 2007}

Letter to the Editor

327 Pulmonary Sarcoidosis Mimicking Metastases in Breast Cancer Patients

Gusakova, I.; Lavrenkov, K.; Ariad, S.; Mermershtain, W. (Beer Sheva)

Review Article

330 Human Chorionic Gonadotropin-Induced Hyperthyroidism in Germ Cell Cancer - a Case Presentation and Review of the Literature Voigt, W.; Maher, G.; Wolf, H.H.; Schmoll, H.J. (Halle)

335 Book Reviews

337 PharmaNews / PharmaTicker

340 Meetings and Conferences

329 Imprint

342 Guidelines for Authors

Forthcoming papers are listed on page 344.

\section{Band 30, Heft 6, Juni 2007}

Brief an die Herausgeber

327 Pulmonale Sarkoidose bei Brustkrebspatientinnen: Leicht zu verwechseln mit Metastasen Gusakova, I.; Lavrenkov, K.; Ariad, S.; Mermershtain, W. (Beer Sheva)

Übersichtsarbeit

330 Durch humanes Choriongonadotropin induzierte Hyperthyreose in Keimzelltumoren - ein Fallbericht und Literaturübersicht

Voigt, W.; Maher, G.; Wolf, H.H.; Schmoll, H.J. (Halle)

335 Buchbesprechungen

337 PharmaNews / PharmaTicker

340 Tagungen und Kongresse

329 Impressum

343 Hinweise für Autoren

Einen Ausblick auf den Inhalt der kommenden Hefte finden Sie auf Seite 344.

\section{KARGER}

Fax +497614520714

E-mail Information@Karger.de www.karger.com
(C) 2007 S. Karger GmbH, Freiburg 